\title{
DOI 10.18699/BGRS/SB-2020-133 \\ Application of ITS1 and ITS2 for population genetic studies of sturgeons (Acipenseridae)
}

Guzel Davletshina, IMCB SB RAS, Novosibirsk, Russia ICG SB RAS, Novosibirsk, Russia guzel@mcb.nsc.ru
Sergey Kliver

IMCB SB RAS, Novosibirsk, Russia skliver@mcb.nsc.ru

Dmitry Prokopoy

ICG SB RAS, Novosibirsk, Russia dprokopov@mcb.nsc.ru
Elena Interesova TSU, Tomsk, Russia e.interesova@ngs.ru

Vladimir Trifonov IMCB SB RAS, Novosibirsk, Russia NSU, Novosibirsk, Russia vlad@mcb.nsc.ru

\begin{abstract}
The order Acipenseridae is a very interesting group for evolutionary genetics: all species have unique morphology, inter-specific hybrids are widely occurring and there are variations between species in ploidy levels. Most acipenserids are endangered due to poaching and special efforts are required for the maintenance of natural populations. The genetic studies of acipenserids are still limited, although these are needed for successful farming. ITS - is the DNA spacer located between the small subunit and large subunit rRNA genes. The genes encoding ribosomal RNAs are located one after another in tandem and are repeated several hundred times, so we use new generation sequencing to estimate the frequency of occurrence of SNPs in the genome of one organism. ITS1 and ITS2 are used as phylogenetic markers to study the relationships between highly diverged taxonomic groups [1]. Despite high interest to different sturgeon species, acipenserid ITS1 and ITS2 sequences are missing in the GenBank depository, and most sturgeon population studies are performed using mitochondrial markers. Here we study the structure of ITS1 and ITS2 in several sturgeon species and demonstrate efficiency of these nuclear markers for species identification and interspecific hybrids confirmation.
\end{abstract}

Keywords - acipenser, phylogenetic markers, ITS, NGS, polyploidy, interspecific hybrids.

Motivation and aim

Motivation

ITS1 and ITS2 are phylogenetic markers, widely used for many species of animals, plants, fungi and bacteria, but these important nuclear markers have never been applied for sturgeons. As stugreons are paleopolyploids, their microsatellite analysis is complicated, and mitochondrial markers characterize only maternal lineages.

Aim

Here we study the structure of ITS1 and ITS2 in Acipenser baerii, A. ruthenus and identify SNPs specific to different species and their populations. Besides, we demonstrate that this nuclear marker facilitates identification of interspecific hybrids.

\section{Methods}

Primers for PCR amplification of sturgeon ITS1 and ITS2 were designed using genome sequence of A.ruthenus. Obtained PCR products from 21 individual were then sequenced on the Illumina platform. Raw sequencing data were preprocessed using custom pipeline followed by variant calling by Samtools.
Results

We generated consensus sequences of ITS1 and ITS2 for sturgeon species: A.baerii and A.ruthenus. We found six single nucleotide substitutions differentiating these two sturgeon species. We sequenced ITS1 and ITS2 of interspecific hybrids between A.baerii and A.ruthenus, and found that indeed there were SNPs in the sequence characteristic for both ITS1 and ITS2. Based on NGS data analysis, we demonstrated that A.ruthenus specific SNPs were represented in around $33 \%$ reads, while A.baerii specific SNPs were found in 67\%, which corresponds to firgt generation hybrids subgenome ratio (as Siberian sturgeon genome is twice as large as sterlet genome) (Table 1).

Table 1. The mean frequency of variant occurrence in the reads for all samples characteristic of ITS1 and ITS2 SNPs in two species of sturgeons and their hybrids

\begin{tabular}{|c|c|c|c|c|c|c|c|}
\hline \multicolumn{2}{|c|}{$\begin{array}{l}\text { Species, } \\
\text { number of } \\
\text { individuals }\end{array}$} & \multicolumn{2}{|c|}{$\begin{array}{l}\text { A. baerii*, } \\
\mathrm{n}=7\end{array}$} & \multicolumn{2}{|c|}{$\begin{array}{l}\text { A. } \text { ruthenus*, } \\
\mathrm{n}=11\end{array}$} & \multicolumn{2}{|c|}{$\begin{array}{l}\text { A.baerii+A.ruthenus, } \\
\mathrm{n}=3\end{array}$} \\
\hline \multicolumn{2}{|c|}{$\begin{array}{l}\text { number of } \\
\text { chromosomes }\end{array}$} & \multicolumn{2}{|l|}{240} & \multicolumn{2}{|l|}{120} & \multicolumn{2}{|l|}{$120+60$} \\
\hline \multirow[t]{2}{*}{ ITS1 } & 1934 & $G(0,974)$ & $\mathrm{A}(0,025)$ & $\mathrm{G}(0,005)$ & $\mathbf{A}(0,995)$ & $\mathrm{G}(0,668)$ & $\mathrm{A}(0,332)$ \\
\hline & 2454 & $\mathbf{T}(\mathbf{0 , 9 7 4 )}$ & $\mathrm{C}(0,025)$ & $\mathrm{T}(0,021)$ & $\mathrm{C}(\mathbf{0 , 9 7 9 )}$ & $\mathrm{T}(0,682)$ & $\mathrm{C}(0,318)$ \\
\hline \multirow[t]{4}{*}{ ITS2 } & 2898 & \begin{tabular}{|l|}
$T(0,747)$ \\
\end{tabular} & $\mathrm{C}(0,253)$ & $\mathrm{T}(0,002)$ & $\begin{array}{l}\mathrm{C}(0,998) \\
\end{array}$ & $\mathrm{T}(0,481)$ & $\mathrm{C}(0,519)$ \\
\hline & 2917 & \begin{tabular}{|l|}
$\mathrm{C}(0,972)$ \\
\end{tabular} & $\mathrm{T}(0,028)$ & $\mathrm{C}(0,002)$ & \begin{tabular}{|l|}
$T(0,998)$ \\
\end{tabular} & $\mathrm{C}(0,655)$ & \begin{tabular}{|l|}
$\mathrm{T}(0,345)$ \\
\end{tabular} \\
\hline & 3065 & \begin{tabular}{|l|}
$T(0,952)$ \\
\end{tabular} & $\mathrm{C}(0,048)$ & $\mathrm{T}(0,002)$ & $\mathrm{C}(0,998)$ & $\mathrm{T}(0,579)$ & $\mathrm{C}(0,421)$ \\
\hline & 3103 & $\mathrm{~T}(\mathbf{0 , 9 7 0 )}$ & $\mathrm{C}(0,030)$ & $\mathrm{T}(0,042)$ & $\mathrm{C}(0,958)$ & $\mathrm{T}(0,625)$ & $\mathrm{C}(0,375)$ \\
\hline
\end{tabular}

parental species were detected in hybrids.

\section{Acknowledgment}

The work was supported by the Russian Science Foundation grant No. 18-44-04007, Guzel Davletshina was additionally funded by the budget project No. 0259-20190003-C-01

\section{References}

[1] Allard M.W. and Honeycutt R.L. (1991) Ribosomal DNA Variation Within and Between Species of Rodents, with Emphasis on the Genus Onychomys. Mol. Biol. Evol. 8: 71-84. 